\title{
Image Quality Assessment Using the Joint Spatial/Spatial-Frequency Representation
}

\author{
Azeddine Beghdadi ${ }^{1}$ and Răzvan lordache ${ }^{2}$ \\ ${ }^{1}$ L2TI-Institute Galilée, Université Paris 13, 93430 Villetaneuse, France \\ ${ }^{2}$ GE Healthcare Technologies, 78530 Buc, France
}

Received 9 December 2004; Revised 20 December 2005; Accepted 9 March 2006

Recommended for Publication by Gonzalo Arce

This paper demonstrates the usefulness of spatial/spatial-frequency representations in image quality assessment by introducing a new image dissimilarity measure based on 2D Wigner-Ville distribution (WVD). The properties of 2D WVD are shortly reviewed, and the important issue of choosing the analytic image is emphasized. The WVD-based measure is shown to be correlated with subjective human evaluation, which is the premise towards an image quality assessor developed on this principle.

Copyright @ 2006 Hindawi Publishing Corporation. All rights reserved.

\section{INTRODUCTION}

Wigner-Ville distribution (WVD) has been proved to be a powerful tool for analyzing the time-frequency characteristics of nonstationary signals [1]. It is well established that WVD-based signal analysis methods overcome the shortcomings of the traditional Fourier-based methods and that it achieves high resolution in both domains.

While WVD is widely used in applications involving 1D signals, the extension to multidimensional signals, in particular to $2 \mathrm{D}$ images has not reached a similar development [2]. The use of WVD for image processing was first suggested by Jacobson and Wechsler [3]. It was shown that WVD is a very efficient tool for capturing the essential nonstationary image structures $[4,5]$. The interesting properties of joint spatial/spatial-frequency representations of images led to other applications of WVD to image processing, in particular in image segmentation [6-10], demonstrating that WVD-based methods provide high discriminating power for signal representation. Indeed, WVD extracts the intrinsic local spectral features of an image. On the basis of this knowledge, the motivation behind the idea of using WVD for image quality measure is that the extraction and evaluation of a distortion in a given image could be expressed as a segmentation problem.

This paper proposes the application of the WVD in analyzing and tracking image distortions for computing an image quality measure. The properties of the $2 \mathrm{D}$ WVD and some implementation aspects are briefly discussed.
With the increasing use of digital video compression and transmission systems, image quality assessment has become a crucial issue. In the last decade, there have been proposed numerous methods for image distortion evaluation inspired from the findings on human visual system (HVS) mechanisms [11]. In the vision research community, it is generally acknowledged that the early visual processing stages involve the creation of a joint spatial/spatial-frequency representation [12]. This motivates the use of the WVD as a tool for analyzing the effects induced by applying a distortion to a given image.

Depending on the required information regarding the original (nondistorted) image quality assessment techniques can be grouped into three classes: the full-reference (FR), the reduced reference (RR), and the nonreference (NR), also called blind, approaches. For the FR methods, one needs the original image; to evaluate the quality of the distorted image, whereas RR methods require only a set of features extracted from both the original and the degraded image. When a priori knowledge on the distortion nature is available and its predictability is well understood, NR measures can be developed, where no information on the image reference is needed.

Straightforward FR objective measures have been proposed in the literature such as PSNR or weighted PSNR [13]. However, such metrics reflect the global properties of the image quality but are inefficient in predicting structural degradations. There is a real need to provide an objective image quality metric consistent with subjective evaluation. Since 
image quality is subjective, the evaluation based on subjective experiments is the most accepted alternative. Unfortunately, subjective image quality assessment necessitates the use of several procedures, which have been formalized by the ITU recommendation [14]. These procedures are complex, time consuming, and nondeterministic. It should be also noticed that perfect correlation with the HVS could never be achieved due to the natural variations in the subjective quality evaluation.

These drawbacks led to the development of other practical and objective measures [11]. Basically, there are two approaches for quantitative image quality measure. The first and more practical approach is the distortion-oriented, like the MSE, PSNR, and other similar measures. However, for this class of distortion measures, the quality metric does not correlate with the subjective evaluation for many types of degradations. The second class corresponds to the HVSmodelling-oriented measures. Unfortunately, there is no satisfying visual perception model that account for all the experimental findings on the HVS. All the proposed models have parameters, which depend on many environment factors and require delicate tuning in order to correlate with the subjective assessment. Recently, a simple and practical measure has been proposed by Wang et al. [15]. This objective measure has been proved to be consistent with the HVS quality assessment for some image degradations. However, this measure is unstable in homogeneous regions.

This paper deals with FR image quality assessment. The simple Wigner-based distortion measure introduced in this paper does not take into account the masking effect. This factor will be introduced in a future work. The comparison of the WVD-based measure with subjective human evaluation and with other objective image quality measures is illustrated through experimental results. This measure could be used for image quality assessment, or as criterion for image coder optimization.

\section{2D WIGNER-VILLE DISTRIBUTION}

The 2D WVD $W_{f}(x, y, u, v)$ of a 2D image $f(x, y)$ assigns to any point $(x, y)$ a 2D spatial-frequency spectrum [6]:

$$
\begin{aligned}
W_{f}(x, y, u, v)= & \int_{\mathbb{R}^{2}} f\left(x+\frac{\alpha}{2}, y+\frac{\beta}{2}\right) \\
& \times f^{*}\left(x-\frac{\alpha}{2}, y-\frac{\beta}{2}\right) e^{-j 2 \pi(\alpha u+\beta v)} d \alpha d \beta,
\end{aligned}
$$

where $x$ and $y$ are the spatial coordinates, $u$ and $v$ are the spatial frequencies, and the asterisk denotes complex conjugation.

The image can be reconstructed up to a sign ambiguity from its WVD:

$$
f(x, y) f^{*}(0,0)=\int_{\mathbb{R}^{2}} W_{f}\left(\frac{x}{2}, \frac{y}{2}, u, v\right) e^{j 2 \pi(x u+y v)} d u d v .
$$

Among the properties of 2D Wigner-Ville distribution, the most important for image processing applications is that it is always a real-valued function and, at the same time, contains the phase information. The 2D Wigner-Ville distribution has many interesting properties related to translation, modulation, scaling, convolution, and localization in spatial/spatial-frequency space, which motivate its use in image analysis applications where the spatial/spatial-frequency features of images are of interest. Actually, the WVD is often thought of as the image energy distribution in the joint spatial/spatial-frequency domain. For a thoughtful description, the reader is referred to [6].

Due to its bilinear nature, the WVD of the sum of two images $f_{1}$ and $f_{2}$ introduces an interference term, usually regarded as undesirable artifacts in image analysis applications. Moreover, as a real image is multicomponent, its WV representation is polluted by interference artifacts and is therefore difficult to interpret [5].

A cleaner spatial/spatial-frequency representation of a real image is obtained by computing the WVD of the associated analytic image, which has such spectral properties $[16,17]$. An analytic image has a spectrum containing only positive (or only negative) frequency components. For a reliable spatial/spatial-frequency representation of the real image, the analytic image should be chosen so that (a) the useful information from the 2D WVD of the real signal is found in the 2D WVD of the analytic image, and (b) the 2D WVD of the analytic image minimizes the interference effect.

In practical applications, the images are of finite support; therefore it is appropriate to apply Wigner analysis to a windowed version of the infinite support images. The effect of the windowing is to smear the WVD representation in the frequency plane only, so that the frequency resolution is decreased but the spatial resolution is unchanged.

Let $f(n, m),(n, m) \in \mathbb{Z}^{2}$ be the discrete image obtained by sampling $f(x, y)$, adopting the convention that the sampling period is normalized to unity in both directions. The following notation is made:

$$
\begin{aligned}
& K(m, n, r, s) \\
& \quad=w(r, s) w^{*}(-r,-s) f(m+r, n+s) f^{*}(m-r, n-s) .
\end{aligned}
$$

The 2D discrete windowed WVD is the straightforward extension of the 1D case presented in [18], and is defined as follows:

$$
W_{f_{w}}\left(m, n, u_{p}, v_{q}\right)=4 \sum_{r=-L}^{L} \sum_{s=-L}^{L} K(m, n, r, s) W_{4}^{r p+s q}
$$

where $N=(2 L+2), W_{4}=e^{-j 4 \pi / N}$, and the normalized spatial-frequency pair is $\left(u_{p}, v_{q}\right)=(p / N, q / N)$. By making a periodic extension of the kernel $K(m, n, r, s)$, for fixed $(m, n)$, (4) can be transformed to match the standard form of a $2 \mathrm{D}$ DFT, except that the twiddle factor is $W_{4}$ instead of $W_{2}$ (see [18] for additional details for $1 \mathrm{D}$ case; the $2 \mathrm{D}$ construction is a direct extension). Thus standard FFT algorithms can be used to calculate the discrete $W_{f_{w}}$. The additional power of two represents a scaling along the frequency axes, and can be neglected in the calculations. 
The properties of the discrete WVD are similar to the continuous WVD, except for the periodicity in the frequency variables, which is one-half the sampling frequency in each direction. Therefore, if $f(x, y)$ is a real image, it should be sampled at twice the Nyquist rate to avoid aliasing effects in $W_{f_{w}}\left(m, n, u_{p}, v_{q}\right)$.

As the real-scene images have rich frequency content, the interference cross-terms may mask the useful components contribution. Therefore a commonly used method to reduce the interference in image analysis applications is to smooth the 2D discrete-windowed WVD in the spatial domain using a smoothing window $h(m, n)$. The price to pay is the spatial resolution reduction. The result is the so-called $2 \mathrm{D}$ discrete pseudo-Wigner distribution (PWD), which, for a symmetric frequency window $(w(r, s)=w(-r,-s))$, is defined as [4]

$$
\begin{aligned}
& P W_{f}\left(m, n, u_{p}, v_{q}\right) \\
& =\sum_{k=-M}^{M} \sum_{\ell=-M}^{M} h(k, \ell) W_{f_{w}}\left(m+k, n+\ell, u_{p}, v_{q}\right) \\
& =4 \sum_{r=-L}^{L} \sum_{s=-L}^{L}|w(r, s)|^{2} W_{4}^{r p+s q} \\
& \quad \times \sum_{k=-M}^{M} \sum_{\ell=-M}^{M} h(k, \ell) f(m+k+r, n+\ell+s) \\
& \times f^{*}(m+k-r, n+\ell-s) .
\end{aligned}
$$

A very important aspect to take into account when using PWD is the choice of $w(r, s)$ and $h(k, \ell)$. The size of the first window, $w(r, s)$, is dictated by the resolution required in the spatial-frequency domain. The spectral shape of the window should be an approximation of the delta function that optimizes the compromise between the central lobe's width and the side lobes' height. A window that complies with these demands is the $2 \mathrm{D}$ extension of Kaiser window, which was used in [4]. The role of the second window, $h(k, \ell)$, is to allow spatial averaging. Its size determines the degree of smoothing. The larger the size is, the lower the spatial resolution becomes. The common choice for this window is the rectangular window.

In the discrete case, there is an additional specific requirement when choosing the analytic image: the elimination of the aliasing effect. Taking into account that all the information of the real image must be preserved in the analytic image, only one analytic image cannot fulfill both requirements. Therefore, either one analytic image is used and some aliasing is allowed or more analytic images are employed which obey two restrictions, (a) the real image can be perfectly reconstructed from the analytic images, and (b) each analytic image is alias-free with respect to WVD. To avoid aliasing, a solution is to use two analytic images, obtained by splitting the region of support of the half-plane analytic image into two equal area subregions $[19,20]$. Although this method requires the computation of two WVD, no aliasing artifacts appear. The WVD of the analytic images can be combined to produce the so-called full-domain PWD [19], which is a spatial/spatial-frequency representation of the real image having the same frequency resolution and support as the original real image. This approach was successfully applied in texture analysis and segmentation in [7].

Employing the analytic images $z_{1}$ and $z_{2}$ described in [20], a full-domain PWD of the real image $f(m, n)$, $F P W_{f}\left(m, n, u_{p}, v_{q}\right)$, can be constructed from $P W_{z_{1}}(m, n$, $\left.u_{p}, v_{q}\right)$ and $P W_{z_{2}}\left(m, n, u_{p}, v_{q}\right)$. In the spatial-frequency domain, the full-domain PWD is, by definition, of periodicity 1 and symmetric with respect to the origin, as the WVD of a real image. It is completely specified by:

$$
\begin{aligned}
& F P W_{f}\left(m, n, u_{p}, v_{q}\right) \\
& \quad=\left\{\begin{array}{l}
P W_{z_{1}}\left(m, n, u_{p}, v_{q}\right), \quad 0 \leq u_{p}<\frac{1}{2}, 0<v_{q}<\frac{1}{2}, \\
P W_{z_{2}}\left(m, n, u_{p}, v_{q}\right), \quad 0 \leq u_{p}<\frac{1}{2}, 0>v_{q} \geq-\frac{1}{2},
\end{array}\right. \\
& F P W_{f}\left(m, n, u_{p}, 0\right) \\
& \quad=P W_{z_{1}}\left(m, n, u_{p}, 0\right)+P W_{z_{2}}\left(m, n, u_{p}, 0\right), \quad 0 \leq u_{p}<\frac{1}{2},
\end{aligned}
$$

Figure 1 illustrates the construction of the full-domain PWD from the PWDs of the single-quadrant analytic images. The same shading identifies identical regions, and the letters are used to follow the mapping of frequency regions of the real image. For instance, the region labeled $A$ in (f) represents the mapping of the region $A$ in the real image spectrum (a) on the spatial-frequency domain of the full-domain PWD. A potential drawback of this approach is that the additional sharp filtering boundaries may introduce ringing effects.

\section{AN IMAGE DISSIMILARITY MEASURE BASED ON 2D WIGNER-VILLE DISTRIBUTION}

It is well known that distortion like a regular pattern or a spike is more visible than distortion "diluted" through the image. Between two distortions with the same energy, that is, same peak signal-to-noise-ratio (PSNR), the more disturbing is the one having a peaked energy distribution in spatial/spatial-frequency plane. The "annoying" distortions are usually highly concentrated in the spatial/spatialfrequency domain. Therefore it seems promising to analyze the quality of a distorted image by looking at its energy distribution in the joint spatial/spatial-frequency domain.

In terms of the effect on the WVD, the noise added to an image influences not only the coefficients in the positions where the noise has nonzero WVD coefficients, but 


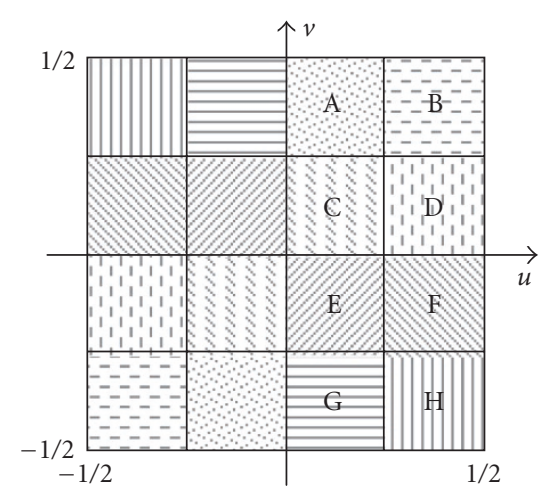

(a)

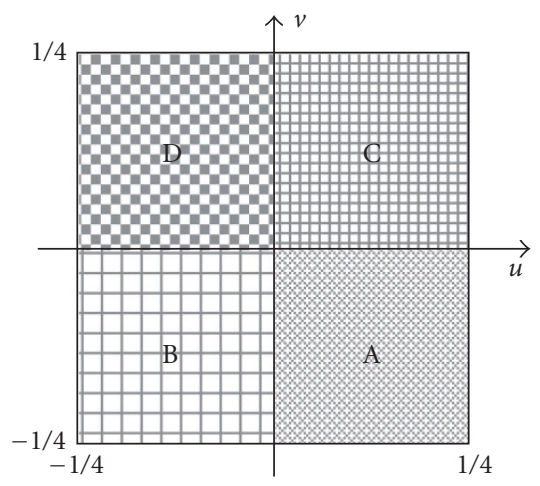

(d)

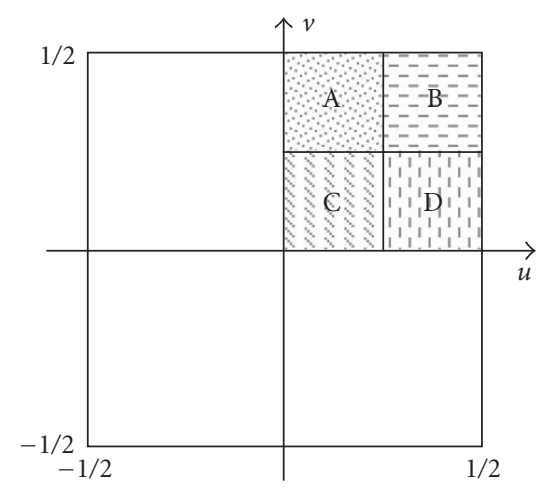

(b)

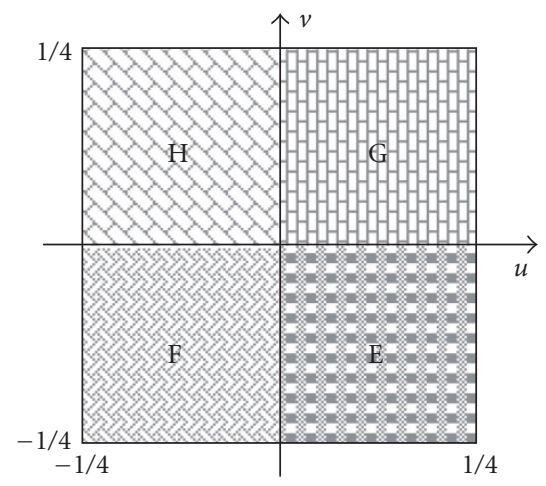

(e)

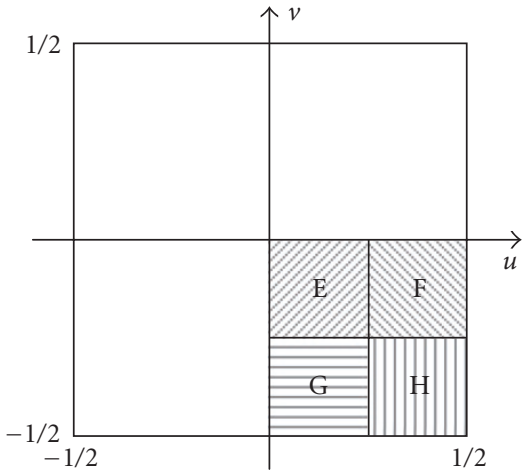

(c)

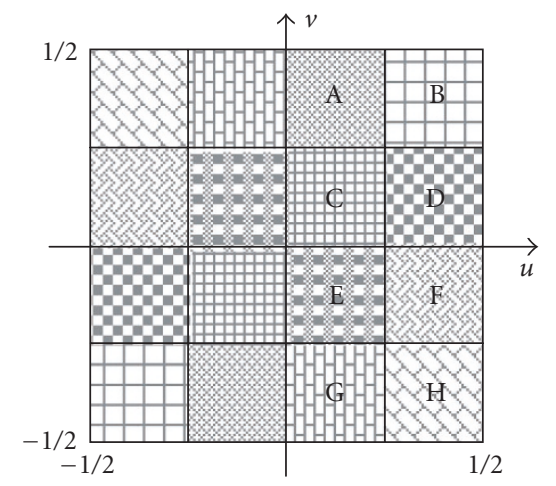

(f)

FIGURE 1: Full-domain WVD computation using a single-quadrant analytic image pair. (a) Spectrum of the real image. (b) Spectrum of the upper-right-quadrant analytic image. (c) Spectrum of the lower-right-quadrant analytic image. (d) Spatial-frequency support of WVD of (b). (e) Spatial-frequency support of WVD of (c). (f) Spatial-frequency support of the full-domain WVD obtained from (d) and (e).

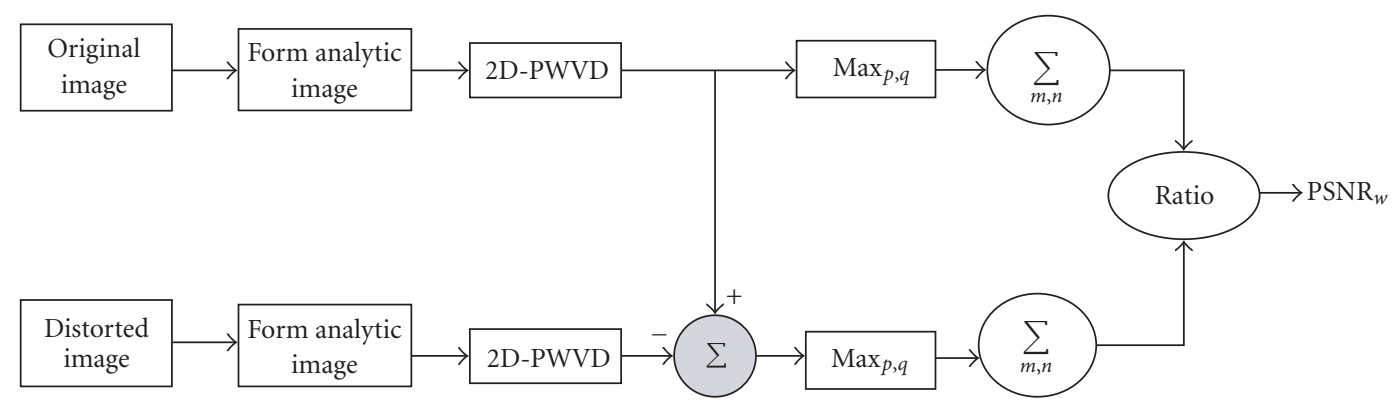

FIgURE 2: Construction of PSNR .

also induces cross-interference terms. The stronger the noise WVD coefficients are, the more important the differences between the noisy image WVD and original image WVD become.
The image quality metric proposed herein is an alternative based on the WVD to the classical PSNR. WVD-based PSNR of a distorted version $g(m, n)$ of the original discrete image $f(m, n)$ is defined as (see Figure 2)

$$
\operatorname{PSNR}_{W}=10 \log _{10} \frac{\sum_{m} \sum_{n} \max _{p, q}\left(\left|F P W_{f}\left(m, n, u_{p}, v_{q}\right)\right|\right)}{\sum_{m} \sum_{n} \max _{p, q}\left(\left|F P W_{f}\left(m, n, u_{p}, v_{q}\right)-F P W_{g}\left(m, n, u_{p}, v_{q}\right)\right|\right)} .
$$




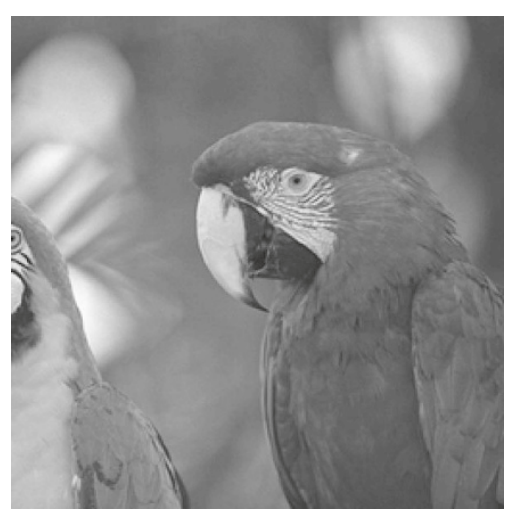

(a) $f$

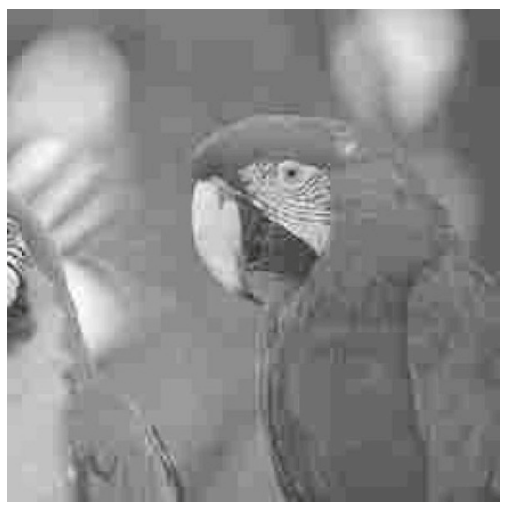

(c) $g_{2}\left(\mathrm{PSNR}=23.74, \mathrm{PSNR}_{W}=17.66\right)$

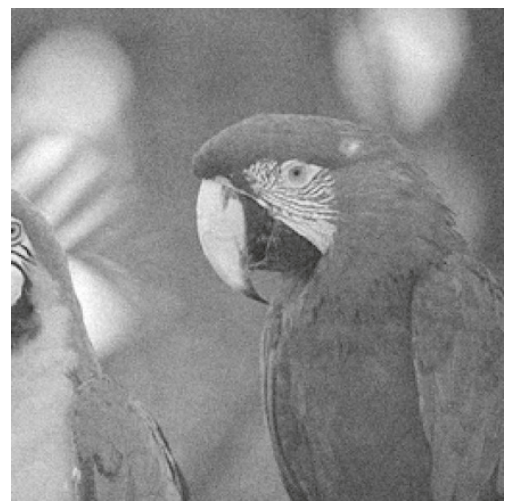

(b) $g_{1}\left(\mathrm{PSNR}=23.70, \mathrm{PSNR}_{W}=21.70\right)$

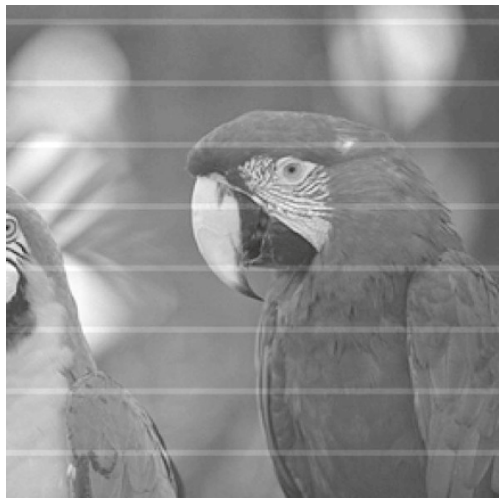

(d) $g_{3}\left(\mathrm{PSNR}=23.70, \mathrm{PSNR}_{W}=14.07\right)$

FIGURe 3: Distorted versions of $256 \times 256$ pixel Parrot image, $f: g_{1}$ is obtained by adding white Gaussian noise on $f$; $g_{2}$ is a JPEG reconstruction of $f$, with a quality factor of $88 ; g_{3}$ is the result of imposing a grid-like interference over $f$. The PSNR and PSNR $W$ values are given in dB.

The 4D PWD reduces to a $2 \mathrm{D}$ function of spatial-frequency variables, which can be interpreted as the local spatial-frequency spectrum of the image at that point. So with the 2D PWD, a local spatial form in an image can be related to some spatial-frequency characteristics in the transform domain.

The use of maximum difference power spectrum as a nonlinearity transformation is motivated and inspired by some findings on the nonlinearity of the HVS. Similar transformations have been successfully used to model intracortical inhibition in the primary visual cortex in an HVS-based method for texture discrimination [21].

For each position $(m, n)$, the highest energy WVD component is retained, as if the contribution of the other components are masked by it. Of course, the masking mechanisms are much more complex, but this coarse approximation leads to results which are more correlated to the HVS perception than PSNR. Among the masking models available in the literature, there is no one single model that takes into account for all masking phenomena in HVS. Nevertheless, there are well-established masking models $[22,23]$ that require a band limited decomposition of the visual signal, so they cannot be directly applied to the current approach. Their adaptation to the WVD representation is a difficult challenge.

Let $\eta_{1}$ and $\eta_{2}$ be two degradations having the same energy. The first, $\eta_{1}$, is additive white Gaussian noise, and the second, $\eta_{2}$, is an interference pattern. While the energy of the noise is evenly spread in the spatial/spatial-frequency plane, the energy of the structured degradation is concentrated in the frequency band of the interference. Thus the WVD of $\eta_{2}$ contains terms which have absolute values larger than any term of WVD of $\eta_{1}$, as the two degradations have the same energy. These peak terms induce larger local differences between WVD of $g_{2}=f+\eta_{2}$ and WVD of $f$, which are captured by "max" operation in the denominator of (7) and lead to a smaller $\mathrm{PSNR}_{W}$ for $g_{2}$.

\subsection{Results and discussion}

To show the interest of the proposed image distortion measure as compared to the PSNR, two examples are presented: Figure 3 illustrates a $256 \times 256$ pixel image and its degraded versions by additive white noise, by an interference pattern, and, respectively, by JPEG coding-decoding, yielding almost 


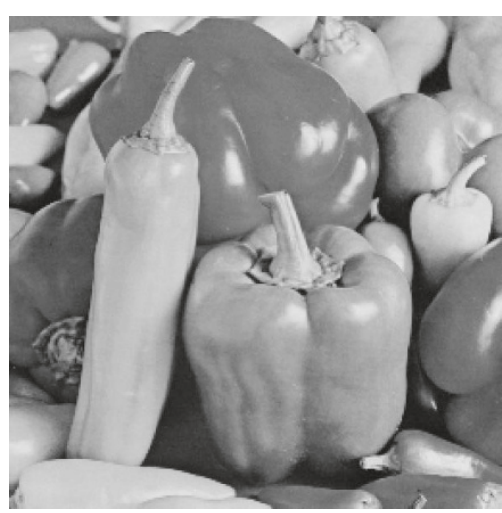

(a) $f$

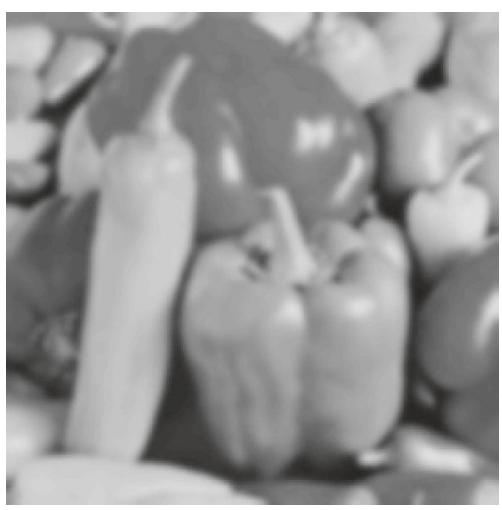

(c) $g_{2}\left(\mathrm{PSNR}=19.83, \mathrm{PSNR}_{W}=14.64\right)$

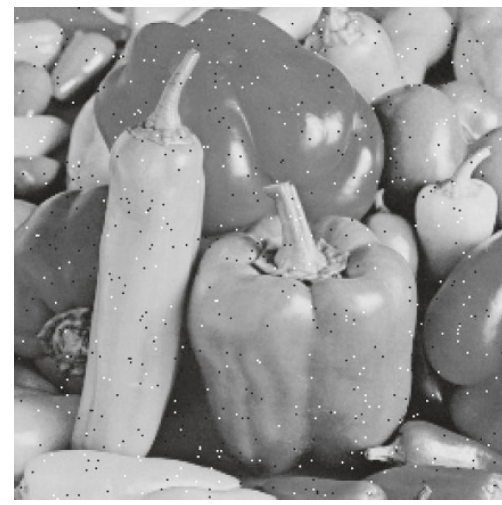

(b) $g_{1}\left(\mathrm{PSNR}=19.81, \mathrm{PSNR}_{W}=18.61\right)$

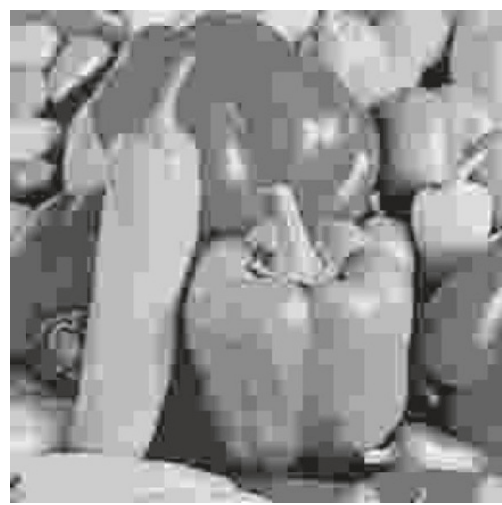

(d) $g_{3}\left(\mathrm{PSNR}=19.85, \mathrm{PSNR}_{W}=12.79\right)$

FIgUre 4: Distorted versions of $256 \times 256$ pixels Peppers image, $f: g_{1}$ is obtained by adding "salt and pepper" noise, $g_{2}$ is a blurred version, and $g_{3}$ is a JPEG reconstruction. The PSNR and PSNR $W$ values are given in $\mathrm{dB}$.

TABLE 1: Observer ranking and image quality metrics for the distorted versions of Parrot image in Figure 3.

\begin{tabular}{lccc}
\hline & Gaussian noise & JPEG & Grid pattern \\
\hline Observer ranking & 1 & 2 & 3 \\
PSNR [dB] & 23.70 & 23.74 & 23.70 \\
SSIM index & $70 \%$ & $82.8 \%$ & $87 \%$ \\
PSNR $_{W A V}[\mathrm{~dB}]$ & 30.63 & 29.33 & 25.75 \\
PSNR $_{W}[\mathrm{~dB}]$ & 21.70 & 17.66 & 14.07 \\
\hline
\end{tabular}

the same PSNR; in Figure 4, a second $256 \times 256$ pixel image and its corrupted versions by "salt and pepper" noise, by blurring, and, respectively, by JPEG coding-decoding, yielding almost the same PSNR.

In both cases, five nonexpert readers were asked to rank the images (including the original) in decreasing order of perceived quality. All readers gave the same ranking, with the original image on top position ( rank 0). The ranking for the distorted images is presented in Table 1 for Parrot image and in Table 2 for Peppers image, together with the WVD-based distortion measure. In both examples, the WVD-based dis-
TABLE 2: Observer ranking and image quality metrics for the distorted versions of Peppers image in Figure 4.

\begin{tabular}{lccc}
\hline & "Salt and pepper" noise & Blur & JPEG \\
\hline Observer ranking & 1 & 2 & 3 \\
PSNR [dB] & 19.80 & 19.83 & 19.84 \\
SSIM index & $80.9 \%$ & $80.6 \%$ & $72.2 \%$ \\
PSNR $_{\text {WAV }}[\mathrm{dB}]$ & 23.45 & 21.74 & 21.32 \\
PSNR $_{W}[\mathrm{~dB}]$ & 18.61 & 14.64 & 12.79 \\
\hline
\end{tabular}

tortion measure is correlated with the subjective quality evaluation.

For the example shown in Figure 3, the observers prefer the white noise distorted image to the interference-perturbed image and to the JPEG-coded image. The reason is that for random degradation, the noise has the same effect in the entire spatial-frequency plane. Therefore, the maximum spectral difference at almost any spatial position is lower than the just noticeable perceptual difference. On the other hand, when the distortion is localized (as interference patterns or distortion induced by JPEG coding), the maximum spectral 
difference corresponding to an important proportion of the pixels has a significant value, much larger than the just noticeable perceptual difference.

Regarding the images in Figure 4, the ordering provided by the observers, from highest to poorest visual quality, corresponds to ranking the images in decreasing order of $\operatorname{PSNR}_{W}$. As for the additive white noise, the power of "salt and pepper" noise is evenly spread over the entire spatialfrequency plane, and the maximum spectral difference at almost any spatial position is lower than the just noticeable perceptual difference. As blurring corresponds to low-pass filtering, the spectral differences between the original (see Figure 4(a)) and the blurred image (see Figure 4(b)) are important at high frequencies, where the signal power is weaker.

For comparison, the wavelet-based PSNR (see [24]), PSNR $_{W A V}$, and the structural similarity index (see [15]), SSIM, are computed in Tables 1 and 2. The SSIM is in contradiction with the observer rating for Figure 3 and in agreement for Figure 4 . The PSNR ${ }_{W A V}$ is correlated with the observer rating for the two examples, but $\mathrm{PSNR}_{W}$ is better in discriminating the image quality of the distortions.

When computing the PSNR ${ }_{W A V}$, one should perform the nonlinear $(\max )$ operation at the different scales, making the measure scale-dependent as expected from the HVS point of view. Moreover, it is pointed out in [24], that the choice of the wavelets, like, for example, biorthogonal 9/7 wavelets against cubic spline wavelets, affects the behavior of the PSNR ${ }_{W A V}$.

Regarding the SSIM, it is known that this measure is unstable in homogeneous regions [15]. Moreover, the SSIM does not take into account the frequency content of the image which plays an important role in the discrimination between spatial structures.

Herein, the objective is to propose an alternative to the standard PSNR, which is independent of the observation distance (and of the observer, in general). Another reason for using the WVD is its perfect spatial-frequency resolution and localization in the joint spatial/spatial-frequency space, so that all frequencies and all location can be analyzed independently, respectively. Furthermore, in contrast to the wavelet transform, the WVD does not require a scale-window function.

One of the main trade-offs of using this type of joint representation is the high dimensionality of the data to be processed. This may prevent the VWD-based measure to be applied to real-time applications, like video quality control, but is of no concern for off-line processes such as comparing still image compression methods or noise filtering methods. Nevertheless, efficient algorithms for computing the WVD are already available $[18,25]$. Moreover, it is the authors' belief that a fast implementation of the WVD is possible by using the huge computational power of the state-of-the-art graphic cards.

\section{SUMMARY AND CONCLUSIONS}

This paper considers the 2D WVD in the framework of image analysis. The advantages and drawbacks of this spatial/ spatial-frequency analysis tool are recalled in the light of some pioneer and recent works in this field.
The usefulness of the WVD in image analysis is demonstrated by considering a particular application, namely, distortion analysis. In this respect, a new image distortion measure is defined. It is calculated using the spatial/spatialfrequency representation of images obtained using 2D WVD. The efficiency of this measure is validated through experiments and informal visual quality assessment tests. It is shown that this measure represents a promising tool for objective measure of image quality, although the masking mechanisms are neglected. To improve the reliability and the performance of the proposed method, a refinement to include a masking model is imperatively needed.

It can be concluded that, taking into consideration some basic, well-established knowledge on the HVS (the joint spatial/spatial-frequency representation, and nonlinear inhibition models), one can develop a simple image distortion measure correlated with the perceptual evaluation.

\section{REFERENCES}

[1] S. Qian and D. Chen, Joint Time-Frequency Analysis: Methods and Applications, Prentice-Hall, Upper Saddle River, NJ, USA, 1994.

[2] B. Boashash, Ed., Time-Frequency Signal Analysis and Processing: A Comprehensive Reference, Elsevier, Oxford, UK, 2003.

[3] L. Jacobson and H. Wechsler, "The Wigner distribution as a tool for deriving an invariant representation of 2-D images," in Proceedings of the International Conference on Pattern Recognition and Image Processing, pp. 218-220, Las Vegas, Nev, USA, June 1982.

[4] T. R. Reed and H. Wechsler, "Segmentation of textured images and Gestalt organization using spatial/spatial-frequency representations," IEEE Transactions on Pattern Analysis and Machine Intelligence, vol. 12, no. 1, pp. 1-12, 1990.

[5] Y. M. Zhu, R. Goutte, and M. Amiel, "On the use of twodimensional Wigner-Ville distribution for texture segmentation," Signal Processing, vol. 30, no. 3, pp. 329-353, 1993.

[6] L. Jacobson and H. Wechsler, "Joint spatial/spatial-frequency representation," Signal Processing, vol. 14, no. 1, pp. 37-68, 1988.

[7] G. Cristóbal and J. Hormigo, "Texture segmentation through eigen-analysis of the Pseudo-Wigner distribution," Pattern Recognition Letters, vol. 20, no. 3, pp. 337-345, 1999.

[8] S. Stankovic, I. Djurovic, and I. Pitas, "Watermarking in the space/spatial-frequency domain using two-dimensional Radon-Wigner distribution," IEEE Transactions on Image Processing, vol. 10, no. 4, pp. 650-658, 2001.

[9] R. Iordache and A. Beghdadi, "A Wigner-Ville distributionbased image dissimilarity measure," in Proceedings of the 6th International Symposium on Signal Processing and Its Applications (ISSPA '01), vol. 2, pp. 430-433, Kuala Lumpur, Malaysia, August 2001.

[10] S. Gabarda and G. Cristóbal, "On the use of a joint spatialfrequency representation for the fusion of multi-focus images," Pattern Recognition Letters, vol. 26, no. 16, pp. 25722578, 2005.

[11] "Special issue on image quality assessment," Signal Processing, vol. 70, 1998.

[12] M. A. García-Pérez and V. Sierra-Vázquez, "Visual processing in the joint spatial/spatial-frequency domain," in Visual Models for Target Detection and Recognition, E. Peli, Ed., pp. 16-62, World Scientific, Hackensack, NJ, USA, 1995. 
[13] A. M. Eskicioglu and P. S. Fisher, "Image quality measures and their performance," IEEE Transactions on Communications, vol. 43, no. 12, pp. 2959-2965, 1995.

[14] ITU-R Recommendation BT.500-7, Methodology for the Subjective Assessment of the Quality of Television Pictures, ITU, Geneva, Switzerland, 1995.

[15] Z. Wang, L. Lu, and A. C. Bovik, "Video quality assessment based on structural distortion measurement," Signal Processing: Image Communication, vol. 19, no. 2, pp. 121-132, 2004, special issue on "Objective video quality metrics".

[16] T. Bülow and G. Sommer, "A novel approach to the $2 \mathrm{~d}$ analytic signal," in Proceedings of the 8th International Conference on Computer Analysis of Images and Patterns (CAIP '99), pp. 2532, Ljubljana, Slovenia, September 1999.

[17] S. L. Hahn, "Multidimensional complex signals with singleorthant spectra," Proceedings of the IEEE, vol. 80, pp. 1287 1300, 1992.

[18] B. Boashash and P. J. Black, "An efficient real-time implementation of the Wigner-Ville distribution," IEEE Transactions on Acoustics, Speech, and Signal Processing, vol. 35, no. 11, pp. 1611-1618, 1987.

[19] J. Homigo and G. Cristobal, "High resolution spectral analysis of images using the pseudo-Wigner distribution," IEEE Transactions on Signal Processing, vol. 46, no. 6, pp. 1757-1763, 1998.

[20] R. Iordache and A. Beghdadi, "Single-quadrant analytic images for 2-D discrete Wigner distribution," in Proceedings of the 8th International Workshop on Systems, Signals and Image Processing (IWSSIP '01), pp. 163-166, Bucharest, Romania, June 2001.

[21] J. Malik and P. Perona, "Preattentive texture discrimination with early vision mechanisms," Journal of the Optical Society of America, vol. 7, no. 5, pp. 923-932, 1990.

[22] A. B. Watson and J. A. Solomon, "Model of visual contrast gain control and pattern masking," Journal of the Optical Society of America A: Optics and Image Science, and Vision, vol. 14, no. 9, pp. 2379-2391, 1997.

[23] P. C. Teo and D. J. Heeger, "Perceptual image distortion," in Proceedings of the 1st IEEE International Conference on Image Processing, vol. 2, pp. 982-986, Austin, Tex, USA, November 1994.

[24] A. Beghdadi and B. Pesquet-Popescu, "A new image distortion measure based on wavelet decomposition," in Proceedings of the 6th International Symposium on Signal Processing and Its Applications (ISSPA '03), vol. 1, pp. 485-488, Paris, France, July 2003.

[25] R. S. Sundaram and K. M. M. Prabhu, "Numerically stable algorithm for computing Wigner-Ville distribution," IEE Proceedings - Vision, Image, and Signal Processing, vol. 144, no. 1, pp. 46-48, 1997.
Azeddine Beghdadi is presently Full Professor at the University of Paris 13 (Institut Galilée) and a Researcher at L2TI laboratory where he does all his research in image and video processing. He obtained his "Maitrise" in physics, and Diplome d'Etudes Approfondies (Master's degree) in optics and signal processing from University Orsay-Paris XI in June 1982 and June 1983, respectively. He also obtained his Ph.D. degree in physics (optics and signal processing) from Uni-

versity Paris 6 in June 1986. He worked at different places including

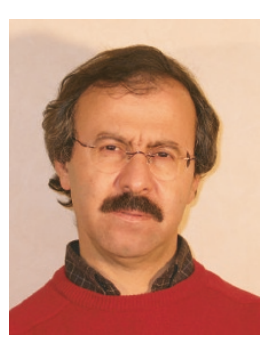

the "Groupe d'Analyse d'Images Biomédicales" (CNAM Paris) and "Laboratoire d'Optique des Solides" (University of Paris 6). From 1987 to 1989, he has been an "Assistant Associé" (Assistant Professor) at University Paris 13. During the period 1987-1998, he was with LPMTM CNRS Laboratory working on scanning electron microscope (SEM) materials image analysis. He published over than one hundred international refereed scientific papers. He is a Funding Member of the L2TI laboratory. His research interests include image quality enhancement and assessment, compression, bio-inspired models for image analysis, and physics-based image analysis. He has served as Conference Chair of ISSPA 2003, and Technical Chair of ISSPA 2005. He also served as session organizer and a Member of the organizing and technical committees for many IEEE conferences. He is Member of IEEE.

Răzvan Iordache received the B.S. degree in electrical engineering and the M.S. degree in biomedical engineering from "Politechnica" University of Bucharest, Romania, and the Ph.D. degree in information technology from Tampere University of Technology, Finland, in 1995, 1996, and 2001, respectively. He is currently a Research Engineer with the Global Diagnostic X-ray Imaging Division, GE Healthcare Technolo-

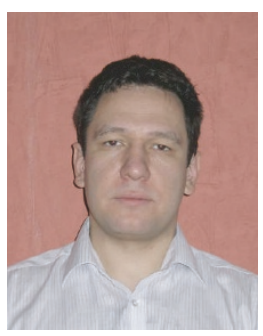
gies, Buc, France. His technical interests are in breast imaging, tomosynthesis, and medical image quality. 